\title{
EXCEPTIONAL BOUNDARY SETS FOR SOLUTIONS OF PARABOLIC PARTIAL DIFFERENTIAL INEQUALITIES
}

\author{
G. N. HILE AND R. Z. YEH
}

\begin{abstract}
Let $\mathscr{M}$ be a second order, linear, parabolic partial differential operator with coefficients defined in a domain $\mathscr{D}=\Omega \times(0, T)$ in $\mathbf{R}^{n} \times \mathbf{R}$, with $\Omega$ a domain in $\mathbf{R}^{n}$. Let $u$ be a suitably regular real function in $\mathscr{D}$ such that $u$ is bounded below and $\mathscr{M} u$ is bounded above in $\mathscr{D}$. If $u \geqslant 0$ on $\Omega \times\{0\}$ except on a set $\Gamma \times\{0\}$, with $\Gamma$ a subset of $\Omega$ of suitably restricted Hausdorff dimension, then necessarily $u \geqslant 0$ also on $\Gamma \times\{0\}$. The allowable Hausdorff dimension of $\Gamma$ depends on the coefficients of $\mathscr{M}$. For example, if $\mathscr{M}$ is the heat operator $\Delta-\partial / \partial t$, the Hausdorff dimension of $\Gamma$ needs to be smaller than the number of space dimensions $n$.

Analogous results are valid for exceptional boundary sets on the lateral boundary, $\partial \Omega \times(0, T)$, of $\mathscr{D}$.
\end{abstract}

1. Introduction. Let $\mathscr{L}$ and $\mathscr{M}$ denote the differential operators given by

$$
\mathscr{L} u:=\sum_{i, j=1}^{n} a_{i j} \frac{\partial^{2} u}{\partial x_{i} \partial x_{j}}+\sum_{i=1}^{n} b_{i} \frac{\partial u}{\partial x_{i}}+c u, \quad \mathscr{M} u:=\mathscr{L} u-\frac{\partial u}{\partial t},
$$

with coefficients defined in some "cylindrical" open set $\mathscr{D}:=\Omega \times(0, T)$, where $\Omega$ is an open subset of $\mathbf{R}^{n}, n \geqslant 2$, and $0<T \leqslant \infty$. The operator $\mathscr{M}$ is assumed to be parabolic in $\mathscr{D}$; in fact, we require that for some positive constants $\Lambda_{0}$ and $\Lambda_{1}$ the eigenvalues of the matrix $\left(a_{i j}\right)$ of second order coefficients, listed in nondecreasing order, satisfy inequalities

$$
0<\Lambda_{0} \leqslant \sum_{i=1}^{n} \lambda_{i}(x, t), \quad 0<\lambda_{1}(x, t) \leqslant \lambda_{n}(x, t) \leqslant \Lambda_{1},
$$

for all $(x, t)$ in $\mathscr{D}$. Let $\Gamma$ be a subset of $\bar{\Omega}$ of Hausdorff dimension less than $\Lambda_{0} / \Lambda_{1}$. Under suitable regularity conditions, we show that if $u$ is a solution in $\mathscr{D}$ of $\mathscr{M} u \leqslant$ constant, with also $u \geqslant$ constant in $\mathscr{D}$, and if $u \geqslant 0$ on $\partial \mathscr{D}-(\Gamma \times\{0\})$, then necessarily $u \geqslant 0$ also on $\Gamma \times\{0\}$.

For example, if the principal part of $\mathscr{L}$, and hence of $\mathscr{M}$, is the Laplace operator, then $\Lambda_{0} / \Lambda_{1}=n$. Then for an equation $\mathscr{M} u=f$, initial values of $u$ on $\bar{\Omega} \times\{0\}$ may be omitted on a set of Hausdorff dimension less than $n$ without affecting the uniqueness of the solution, provided that $u$ and $f$ are assumed bounded.

Received by the editors July 16, 1986 and, in revised form, February 2, 1987.

1980 Mathematics Subject Classification. Primary 35K10; Secondary 35K15, 35K20.

Key words and phrases. Parabolic operator, Phragmén-Lindelöf principle, exceptional boundary set, Hausdorff dimension. 
We have analogous results for lateral boundary exceptional sets, on the part of the boundary $\partial \Omega \times(0, T)$. Here the allowable Hausdorff dimension of the exceptional set depends on the coefficients of $\mathscr{M}$ and also on the geometry of $\partial \Omega$.

These results extend standard versions of so-called "Phragmén-Lindelöf principles". Phragmén-Lindelöf principles generally describe the behavior of a solution of a differential equation or inequality when the solution is known to be nonnegative on the boundary of a domain except at an exceptional boundary point. Under appropriate growth restrictions on the solution, one concludes that the solution must be nonnegative also at the exceptional point.

For elliptic equations Phragmén-Lindelöf principles were first proved by Gilbarg [4] in two dimensions, and by Hopf [9] in general $\mathbf{R}^{n}$. Further refinements and strengthening of these results were given by Serrin [16] and Friedman [3]. Miller [11, 12] obtained Phragmén-Lindelöf results for general uniformly elliptic operators with no continuity at all assumed on the coefficients. He also established existence of maximal and minimal Phragmén-Lindelöf indices for classes of uniformly elliptic operators with eigenvalues of the matrix of second order coefficients contained within a fixed range. Oddson $[13,14]$ derived explicit formulas for these indices in the two-dimensional case. For other discussions of Phragmén-Lindelöf principles see Bear and Hile [1], Fife [2], Habetha [5], Herzog [6], Hile and Yeh [7, 8], Lax [10], and the book of Protter and Weinberger [15].

In [8] the present authors gave a version of the Phragmén-Lindelöf principle for elliptic second order partial differential inequalities where the exceptional boundary set is a set of restricted Hausdorff dimension rather than a single point. This paper gives analogous results for parabolic differential inequalities.

In [3] A. Friedman presented a Phragmén-Lindelöf principle for parabolic differential equations with the exceptional boundary point being the point at infinity.

2. Base points. We again let $\mathscr{L}$ and $\mathscr{M}$ denote the differential operators defined by $(*)$ of the introduction. The coefficients $a_{i j}, b_{i}, c$, for $i, j=1, \ldots, n$, are real valued functions of $(x, t)=\left(x_{1}, \ldots, x_{n}, t\right)$, defined in an open set $\mathscr{D}:=\Omega \times(0, T)$, with $\Omega$ an open subset of $\mathbf{R}^{n}, n \geqslant 2$, and $0<T \leqslant \infty$. The matrix of second order coefficients, $A=\left(a_{i j}\right)$, is assumed symmetric and positive definite in $\mathscr{D}$; thus $\mathscr{M}$ is a parabolic operator, and the eigenvalues of $A$ in $\mathscr{D}$, numbered in nondecreasing order, satisfy $0<\lambda_{1}(x, t) \leqslant \lambda_{2}(x, t) \leqslant \cdots \leqslant \lambda_{n}(x, t)$, for all $(x, t)$ in $\mathscr{D}$. Furthermore, throughout this section we assume that for some positive constants $\Lambda_{0}$ and $\Lambda_{1}$ and for $(x, t)$ in $\mathscr{D}$,

(A) $0<\Lambda_{0} \leqslant \operatorname{trace} A(x, t)=\sum_{i=1}^{n} \lambda_{i}(x, t), \quad 0<\lambda_{1}(x, t) \leqslant \lambda_{n}(x, t) \leqslant \Lambda_{1}$.

The vector $b=\left(b_{1}, \ldots, b_{n}\right)$ of first order coefficients is required to satisfy, for some nonnegative function $b_{0}$ on $(0, T)$ and for $(x, t)$ in $\mathscr{D}$,

$$
|b(x, t)| \leqslant b_{0}(t)=o\left(t^{-1 / 2}\right) \text { as } t \rightarrow 0^{+} .
$$

Finally, we require on the zero order coefficient $c$ the condition, for $(x, t)$ in $\mathscr{D}$,

$$
c(x, t) \leqslant 0 \text {. }
$$


First we prove two preliminary lemmas concerning barrier functions.

LEMMA 1-A. Let $\phi$ be defined on $\mathbf{R}^{n} \times(0, \infty)$ as the function

$$
\phi(x, t):=t^{-\alpha} e^{-\sigma|x|^{2} / t},
$$

where $\alpha$ and $\sigma$ are positive constants chosen so that

$$
0<2 \alpha<4 \sigma \Lambda_{0}<\Lambda_{0} / \Lambda_{1} .
$$

Then there exist positive constants $T_{1}$ and $\gamma_{1}$, depending on $\Lambda_{0}, \Lambda_{1}, b_{0}, T, \alpha, \sigma$, such that for $(x, t)$ in $\Omega \times\left(0, T_{1}\right)$,

$$
\begin{gathered}
\mathscr{M} \phi(x, t) \leqslant-\gamma_{1}\left(t+|x|^{2}\right) t^{-2} \phi(x, t), \\
\left|\frac{\partial \phi}{\partial x_{i}}(x, t)\right| \leqslant \gamma_{1}^{-1} t^{-1}|x| \phi(x, t), \quad i=1, \ldots, n, \\
\left|\frac{\partial^{2} \phi}{\partial x_{i} \partial x_{j}}(x, t)\right| \leqslant \gamma_{1}^{-1} t^{-2}\left(\delta_{i j} t+|x|^{2}\right) \phi(x, t), \quad i, j=1, \ldots, n, \\
\left|\frac{\partial \phi}{\partial t}(x, t)\right| \leqslant \gamma_{1}^{-1} t^{-2}\left(t+|x|^{2}\right) \phi(x, t) .
\end{gathered}
$$

Moreover, for any constant $r>0$, on the paraboloid $t+|x|^{2}=r^{2}, t>0$, we have the inequality,

$$
\phi\left(x, t+r^{2}\right) \geqslant \gamma_{1} r^{-2 \alpha} .
$$

Proof. Calculations yield

$$
\begin{gathered}
\frac{\partial \phi}{\partial x_{i}}=-\frac{2 \sigma}{t} x_{i} \phi, \quad \frac{\partial \phi}{\partial t}=\left(-\frac{\alpha}{t}+\frac{\sigma|x|^{2}}{t^{2}}\right) \phi \\
\frac{\partial^{2} \phi}{\partial x_{i} \partial x_{j}}=\left(-2 \delta_{i j} \frac{\sigma}{t}+\frac{4 \sigma^{2}}{t^{2}} x_{i} x_{j}\right) \phi \\
\mathscr{M} \phi=\left(-\frac{2 \sigma}{t}(\operatorname{trace} A)+\frac{4 \sigma^{2}}{t^{2}} A x \cdot x-2 \sigma \frac{b \cdot x}{t}+c+\frac{\alpha}{t}-\frac{\sigma|x|^{2}}{t^{2}}\right) \phi
\end{gathered}
$$

hence, application of (A), (B), (C) leads to

$$
\mathscr{M}_{\phi} \leqslant\left(-\frac{2 \sigma}{t} \Lambda_{0}+4 \Lambda_{1} \sigma^{2} \frac{|x|^{2}}{t^{2}}+\frac{2 \sigma}{t} b_{0}(t)|x|+\frac{\alpha}{t}-\frac{\sigma}{t^{2}}|x|^{2}\right) \phi .
$$

For any positive constant $\xi$ we have

$$
2 b_{0}(t)|x| \leqslant \xi \frac{|x|^{2}}{t}+\xi^{-1} t b_{0}(t)^{2}
$$

upon setting $\xi=\left(1-4 \sigma \Lambda_{1}\right) / 2$ and observing from (1.2) that $\xi>0$, we obtain from above that

$$
\mathscr{M} \phi \leqslant\left(\alpha-2 \sigma \Lambda_{0}+\frac{2 \sigma t}{1-4 \sigma \Lambda_{1}} b_{0}(t)^{2}\right) t^{-1} \phi-\left(1-4 \sigma \Lambda_{1}\right) \frac{\sigma|x|^{2}}{2 t^{2}} \phi .
$$


Applying now (1.2) and (B), we arrive at (1.3) for $(x, t)$ in $\Omega \times\left(0, T_{1}\right)$ provided that $T_{1}$ and $\gamma_{1}$ are chosen sufficiently small.

Inequalities (1.4)-(1.6) follow from (1.8) and (1.9), again with $\gamma_{1}$ chosen sufficiently small. To obtain (1.7), we observe that for $t+|x|^{2}=r^{2}, t>0$, and for $\gamma_{1}$ sufficiently small,

$$
\begin{aligned}
\phi\left(x, t+r^{2}\right) & =\left(t+r^{2}\right)^{-\alpha} \exp \left[-\sigma|x|^{2} /\left(t+r^{2}\right)\right] \\
& \geqslant\left(r^{2}+r^{2}\right)^{-\alpha} \exp \left[-\sigma\left(r^{2}-t\right) /\left(r^{2}+t\right)\right] \\
& \geqslant\left(2 r^{2}\right)^{-\alpha} e^{-\sigma} \geqslant \gamma_{1} r^{-2 \alpha} .
\end{aligned}
$$

Lemma 1-B. Let $\psi$ be defined on $\mathbf{R}^{n} \times(0, \infty)$ as the function

$$
\psi(x, t):=t^{1-\beta}+\left(1+t^{\beta}\right)|x|^{2},
$$

where $\beta$ is a positive constant, $0<\beta<1$. Then there exist positive constants $T_{2}$ and $\gamma_{2}$, depending on $n, \Lambda_{1}, b_{0}, \beta, T$, such that for $(x, t)$ in $\Omega \times\left(0, T_{2}\right)$,

$$
\begin{gathered}
\mathscr{M} \psi(x, t) \leqslant-\gamma_{2}\left[t^{-\beta}+t^{\beta-1}|x|^{2}\right], \\
\left|\frac{\partial \psi}{\partial x_{i}}(x, t)\right| \leqslant 4|x|, \quad\left|\frac{\partial^{2} \psi}{\partial x_{i} \partial x_{j}}(x, t)\right| \leqslant 4 \delta_{i j}, \\
\left|\frac{\partial \psi}{\partial t}(x, t)\right| \leqslant t^{-\beta}+t^{\beta-1}|x|^{2}
\end{gathered}
$$

ProOF. Calculations yield

$$
\begin{gathered}
\frac{\partial \psi}{\partial x_{i}}=\left(1+t^{\beta}\right)\left(2 x_{i}\right), \quad \frac{\partial \psi}{\partial t}=(1-\beta) t^{-\beta}+\beta t^{\beta-1}|x|^{2}, \\
\frac{\partial^{2} \psi}{\partial x_{i} \partial x_{j}}=2 \delta_{i j}\left(1+t^{\beta}\right), \\
\mathscr{M} \psi=2\left(1+t^{\beta}\right)(\operatorname{trace} A)+2\left(1+t^{\beta}\right) b \cdot x+c \psi-(1-\beta) t^{-\beta}-\beta t^{\beta-1}|x|^{2} ;
\end{gathered}
$$

then application of $(\mathrm{A}),(\mathrm{B}),(\mathrm{C})$ gives

$$
\mathscr{M} \psi \leqslant 2 n \Lambda_{1}\left(1+t^{\beta}\right)+2\left(1+t^{\beta}\right) b_{0}(t)|x|-(1-\beta) t^{-\beta}-\beta t^{\beta-1}|x|^{2} .
$$

We choose $T_{2} \leqslant 1$, which implies $1+t^{\beta} \leqslant 2$; we also use

$$
b_{0}(t)|x| \leqslant \frac{\beta}{8} t^{\beta-1}|x|^{2}+\frac{2}{\beta} t^{1-\beta} b_{0}(t)^{2}
$$

and obtain

$$
\mathscr{M} \psi \leqslant 4 n \Lambda_{1}+\frac{8}{\beta} t^{1-\beta} b_{0}(t)^{2}-(1-\beta) t^{-\beta}-\frac{\beta}{2} t^{\beta-1}|x|^{2} .
$$

But from (B), $t^{1-\beta} b_{0}(t)^{2}=o\left(t^{-\beta}\right)$; thus (1.11) follows for $T_{2}$ and $\gamma_{2}$ chosen sufficiently small.

Inequalities (1.12), (1.13) are consequences of (1.14), (1.15) and the restriction $0<t \leqslant T_{2} \leqslant 1$. 
If $z$ is a point on $\partial \mathscr{D}$ and $u$ is a real valued function in $\mathscr{D}$, by the phrase " $u \geqslant 0$ at $z$ " we mean that $\lim \inf u(x, t) \geqslant 0$ as $(x, t)$ approaches $z$ from inside $\mathscr{D}$. If $E$ is a subset of $\partial \mathscr{D}$, by " $u \geqslant 0$ on $E$ " we mean that $u \geqslant 0$ at every point $z$ on $E$. If $u \geqslant 0$ on the intersection of $\partial \mathscr{D}-E$ with a neighborhood of $z$ (or of $E$ ), then we say " $u \geqslant 0$ on $\partial \mathscr{D}-E$ near $z$ " (or, resp., near $E$ ).

We denote by $\mathscr{H}(E)$ the Hausdorff dimension of a set $E$. We require the following elementary consequence of the definition of Hausdorff dimension. Its brief justification appears in [8].

Lemma 1-C. Let $E$ be a subset of $\mathbf{R}^{n}$ with Hausdorff dimension $\mathscr{H}(E)$. If $\mu$ is a positive number such that $\mathscr{H}(E)<\mu$, then for any $\varepsilon>0$ and $\nu>0$ there exists $a$ countable covering of $E$ by open balls $\left\{B_{i}\right\}$ in $\mathbf{R}^{n}$ such that the center of each $B_{i}$ is $a$ point of $E$, each radius $r_{i}$ of $B_{i}$ satisfies $0<r_{i} \leqslant \nu$, and moreover $\sum_{i} r_{i}^{\mu}<\varepsilon$.

THEOREM 1. Assume conditions (A), (B), (C) hold on the coefficients of the operator $\mathscr{M}$ as defined by (*). Let $\Gamma$ be a subset of $\bar{\Omega}$, with

$$
0 \leqslant \mathscr{H}(\Gamma)<\Lambda_{0} / \Lambda_{1},
$$

and let $y_{0}$ be a point on $\Gamma$. Let $u$ be a real valued function in $\mathscr{D}=\Omega \times(0, T)$, of class $C^{2}$ with respect to $x$ and of class $C^{1}$ with respect to $t$. Suppose that for some constants $M, \tau$, with $M \geqslant 0,0<\tau<1$, we have for $(x, t)$ in $\mathscr{D}$,

$$
u(x, t) \geqslant-M, \quad \mathscr{M} u(x, t) \leqslant M t^{-\tau} ;
$$

then

$$
u \geqslant 0 \quad \text { on } \partial \mathscr{D}-(\Gamma \times\{0\}) \text { near }\left(y_{0}, 0\right)
$$

implies

$$
u \geqslant 0 \text { at }\left(y_{0}, 0\right) .
$$

Proof. We may translate coordinates, if necessary, so that $y_{0}=0$ in $\mathbf{R}^{n}$. Let $\psi$ be a barrier function as described in Lemma 1-B, where $\beta$ is chosen so that $0<\tau<\beta$ $<1$. Let $\delta$ be the positive constant $\delta:=\left[\Lambda_{0} / \Lambda_{1}-\mathscr{H}(\Gamma)\right] / 2$; then choose positive constants $\alpha$, $\sigma$ so that

$$
0 \leqslant \mathscr{H}(\Gamma)=\Lambda_{0} / \Lambda_{1}-2 \delta<\Lambda_{0} / \Lambda_{1}-\delta<2 \alpha<4 \sigma \Lambda_{0}<\Lambda_{0} / \Lambda_{1}
$$

and let $\phi$ be a barrier function as described in Lemma 1-A. By choosing $T_{1}$ and/or $T_{2}$ smaller, if necessary, and then $T$ also smaller, we may arrange the relation $T_{1}=T_{2}=T \leqslant 1$.

Condition (1.18) implies the existence of an open ball $\mathscr{B}$ in $\mathbf{R}^{n+1}$, centered at 0 , with radius $\mathscr{B}<T \leqslant 1$, such that

$$
u \geqslant 0 \text { on } \overline{\mathscr{B}} \cap[\partial \mathscr{D}-(\Gamma \times\{0\})] .
$$

(The set $\overline{\mathscr{B}} \cap \partial \mathscr{D}$ may include part of the lateral boundary, $\partial \Omega \times[0, T]$, of $\mathscr{D}$; in fact, it is possible that $y_{0}=0 \in \partial \Omega$.) Conditions (1.11) and (1.17) imply that in $\mathscr{B} \cap \mathscr{D}$,

$$
\mathscr{M} u(x, t)+\mathscr{M} \psi(x, t) \leqslant M t^{-\tau}-\gamma_{2} t^{-\beta}=\left(M t^{\beta-\tau}-\gamma_{2}\right) t^{-\beta}
$$


Since $\beta>\tau$, we may choose $\mathscr{B}$ smaller, if necessary, so that also

$$
\mathscr{M}(u+\psi)<0 \text { in } \mathscr{B} \cap \mathscr{D} \text {. }
$$

We will establish (1.19) by showing that $\lim \inf u(x, t) \geqslant 0$ as $(x, t) \rightarrow(0,0)$ in $\mathscr{B} \cap \mathscr{D}$.

Denote the radius of $\mathscr{B}$ by $r$; recall that $0<r<T \leqslant 1$. Let $\nu$ be a positive constant sufficiently small that

$$
0<\nu<r, \quad r+\nu^{2}<T, \quad \gamma_{1} \nu^{\Lambda_{0} / \Lambda_{1}-\delta-2 \alpha}>M .
$$

(Since $\Lambda_{0} / \Lambda_{1}-\delta-2 \alpha<0$, the last condition is attainable.) We have $\mathscr{H}(\Gamma)<$ $\Lambda_{0} / \Lambda_{1}-\delta$; thus for any $\varepsilon>0$, Lemma 1-C confirms the existence of a countable covering of $\Gamma$ by open balls $\left\{B_{i}\right\}$ in $\mathbf{R}^{n}$, such that the center of each $B_{i}$, say $y_{i}$, lies on $\Gamma$, the radius of each $B_{i}$, say $r_{i}$, satisfies $0<r_{i} \leqslant \nu$, and moreover so that

$$
\sum_{i} r_{i}^{\Lambda_{0} / \Lambda_{1}-\delta}<\varepsilon
$$

We define a function $v$ in $\mathscr{B} \cap \mathscr{D}$ according to

$$
\begin{aligned}
v(x, t):= & u(x, t)+\left(1+M / r^{2}\right) \psi(x, t) \\
& +\sum_{i} r_{i}^{\Lambda_{0} / \Lambda_{1}-\delta} \phi\left(x-y_{i}, t+r_{i}^{2}\right) .
\end{aligned}
$$

Observe from (1.23) that $t+r_{i}^{2} \leqslant t+\nu^{2} \leqslant r+\nu^{2}<T$ in $\mathscr{B} \cap \mathscr{D}$; thus each function $\phi\left(x-y_{i}, t+r_{i}^{2}\right)$ is defined for $(x, t)$ in $\mathscr{B} \cap \mathscr{D}$, and moreover will satisfy appropriately "translated" versions of (1.3)-(1.6). In fact, the estimates (1.4)-(1.6), (1.24), along with the estimate $\phi(x, t) \leqslant t^{-\alpha}$, show that the series on the right of (1.25) converges uniformly on compact subsets of $\mathscr{B} \cap \mathscr{D}$, along with the series of space derivatives up to order two and time derivative to order one. Thus the series represents a function in $\mathscr{B} \cap \mathscr{D}$ of class $C^{2}$ in $x$ and class $C^{1}$ in $t$, and the series may be differentiated termwise up to these orders. From (1.3), (1.11), and (1.22) we conclude therefore that in $\mathscr{B} \cap \mathscr{D}$,

$$
\mathscr{M} v \leqslant \mathscr{M} u+\left(1+M / r^{2}\right) \mathscr{M} \psi \leqslant \mathscr{M}(u+\psi)<0 .
$$

Now we define "inverted paraboloids" $\left\{\mathscr{P}_{i}\right\}$, according to

$$
\mathscr{P}_{i}:=\left\{(x, t):\left|x-y_{i}\right|^{2}+t<r_{i}^{2}\right\} .
$$

Since $\mathscr{P}_{i}$ contains the set $B_{i} \times\{0\}$, the collection $\left\{\mathscr{P}_{i}\right\}$ is a covering of $\Gamma \times\{0\}$. Moreover, $t<r_{i}^{2} \leqslant r_{i} \leqslant \nu<1$ in $\mathscr{P}_{i}$. We further define open sets

$$
\hat{\mathscr{B}}:=\mathscr{B} \cap \mathscr{D}, \quad \mathscr{P}:=\bigcup_{i} \mathscr{P}_{i} \text {. }
$$

Since $t \leqslant \nu<r$ in each $\mathscr{P}_{i}$, the open set $\hat{\mathscr{B}}-\overline{\mathscr{P}}$ is nonempty. We will show that $v \geqslant 0$ in $\hat{\mathscr{B}}-\overline{\mathscr{P}}$ by applying the minimum principle to $v$ in this open set.

We divide the boundary of $\hat{\mathscr{B}}-\overline{\mathscr{P}}$ into three parts. Recall that for sets $A, B$ in $\mathbf{R}^{n+1}$

$$
\begin{aligned}
\partial(A \cap B) & \subset(\partial A \cap \bar{B}) \cup(A \cap \partial B) \\
& =(\partial A \cap B) \cup(A \cap \partial B) \cup(\partial A \cap \partial B) ;
\end{aligned}
$$


moreover, $\partial A=\partial\left(A^{c}\right)$ where $A^{c}$ is the complement of $A, \partial \bar{A} \subset \partial A$, and $\partial A \subset A^{c}$ provided $A$ is open. Thus,

$$
\begin{aligned}
\partial(\hat{\mathscr{B}}-\overline{\mathscr{P}}) & =\partial\left(\hat{\mathscr{B}} \cap(\overline{\mathscr{P}})^{c}\right) \subset\left(\partial \hat{\mathscr{B}} \cap(\overline{\mathscr{P}})^{c}\right) \cup(\hat{\mathscr{B}} \cap \partial \overline{\mathscr{P}}) \cup(\partial \hat{\mathscr{B}} \cap \partial \overline{\mathscr{P}}) \\
& \subset(\partial \hat{\mathscr{B}}-\mathscr{P}) \cup(\hat{\mathscr{B}} \cap \partial \mathscr{P}) .
\end{aligned}
$$

Since also,

$$
\partial \hat{\mathscr{B}}=\partial(\mathscr{B} \cap \mathscr{D}) \subset(\partial \mathscr{B} \cap \overline{\mathscr{D}}) \cup(\mathscr{B} \cap \partial \mathscr{D}),
$$

we have

$$
\partial(\hat{\mathscr{B}}-\overline{\mathscr{P}}) \subset(\partial \mathscr{B} \cap \overline{\mathscr{D}}-\mathscr{P}) \cup(\mathscr{B} \cap \partial \mathscr{D}-\mathscr{P}) \cup(\hat{\mathscr{B}} \cap \partial \mathscr{P}) .
$$

We investigate separately these three parts of $\partial(\hat{\mathscr{B}}-\overline{\mathscr{P}})$.

(i) On $\partial \mathscr{B} \cap \overline{\mathscr{D}}-\mathscr{P},(1.25),(1.17),(1.10)$, and $0 \leqslant t \leqslant 1,|x|^{2}+t^{2}=r^{2}$ yield

$$
\begin{aligned}
v(x, t) & \geqslant u(x, t)+\left(M / r^{2}\right) \psi(x, t) \\
& \geqslant-M+\left(M / r^{2}\right)\left(t^{2}+|x|^{2}\right)=0 .
\end{aligned}
$$

(ii) On $\mathscr{B} \cap \partial \mathscr{D}-\mathscr{P}$, we have $v \geqslant 0$ since $u \geqslant 0$ on $\overline{\mathscr{B}} \cap[\partial \mathscr{D}-(\Gamma \times\{0\})]$ and $(\mathscr{B} \cap \partial \mathscr{D}-\mathscr{P}) \subset \overline{\mathscr{B}} \cap[\partial \mathscr{D}-(\Gamma \times\{0\})]$.

(iii) On $\hat{\mathscr{B}} \cap \partial \mathscr{P}$, we have $t>0$; since $t \leqslant r_{i}$ in $\overline{\mathscr{P}}_{i}$ and $r_{i} \rightarrow 0$ as $i \rightarrow \infty$ by (1.24), for each $(x, t)$ in $\hat{\mathscr{B}} \cap \partial \mathscr{P}$ there exists a positive constant $d_{(x, t)}$ such that the distance from $(x, t)$ to $\overline{\mathscr{P}}_{i}$ is larger than $d_{(x, t)}$ for all but a finite number of $i$. Thus for each $(x, t)$ in $\hat{\mathscr{B}} \cap \partial \mathscr{P}$ there exists a positive integer $N$ such that

$$
(x, t) \in \partial\left(\bigcup_{i=1}^{N} \mathscr{P}_{i}\right) \subset \bigcup_{i=1}^{N} \partial \mathscr{P}_{i},
$$

which means that $(x, t) \in \partial \mathscr{P}_{j}$ for some $j$. But then, by (1.25), (1.17), (1.7), (1.23) and $r_{j} \leqslant \nu$,

$$
\begin{aligned}
v(x, t) & \geqslant u(x, t)+r_{j}^{\Lambda_{0} / \Lambda_{1}-\delta_{\phi}}\left(x-y_{j}, t+r_{j}^{2}\right) \\
& \geqslant-M+r_{j}^{\Lambda_{0} / \Lambda_{1}-\delta}\left(\gamma_{1} r_{j}^{-2 \alpha}\right) \\
& \geqslant-M+\gamma_{1} \nu^{\Lambda_{0} / \Lambda_{1}-\delta-2 \alpha} \geqslant 0 .
\end{aligned}
$$

We have now shown that $\mathscr{M} v<0$ in $\mathscr{B} \cap \mathscr{D}$ and $v \geqslant 0$ on

$$
\partial(\hat{\mathscr{B}}-\overline{\mathscr{P}})=\partial(\mathscr{B} \cap \mathscr{D}-\overline{\mathscr{P}}) \text {. }
$$

By the minimum principle for parabolic operators (see [15]), we conclude that $v \geqslant 0$ in $\mathscr{B} \cap \mathscr{D}-\overline{\mathscr{P}}$. But by (1.1) and (1.24), the series in (1.25) can be bounded in $\mathscr{B} \cap \mathscr{D}$ by

$$
\sum_{i} r_{i}^{\Lambda_{0} / \Lambda_{1}-\delta} \phi\left(x-y_{i}, t+r_{i}^{2}\right) \leqslant \sum_{i} r_{i}^{\Lambda_{0} / \Lambda_{1}-\delta}\left(t+r_{i}^{2}\right)^{-\alpha} \leqslant t^{-\alpha} \varepsilon ;
$$

hence from $v \geqslant 0$ it follows that in $\mathscr{B} \cap \mathscr{D}-\overline{\mathscr{P}}$,

$$
u(x, t)+\left(1+M / r^{2}\right) \psi(x, t) \geqslant-\varepsilon t^{-\alpha} .
$$


If we let $\nu \rightarrow 0^{+},(1.23)$ is preserved, and $\mathscr{B} \cap \mathscr{D}-\overline{\mathscr{P}}$ widens to include all of $\mathscr{B} \cap \mathscr{D}$ since $t \leqslant \nu$ in $\mathscr{P}$; since also $\varepsilon$ is arbitrary we conclude that in $\mathscr{B} \cap \mathscr{D}$,

$$
u(x, t)+\left(1+M / r^{2}\right) \psi(x, t) \geqslant 0 .
$$

By (1.10), $\psi(x, t) \rightarrow 0$ as $(x, t) \rightarrow\left(0,0^{+}\right)$; thus $\lim \inf u(x, t) \geqslant 0$ as $(x, t) \rightarrow 0$ in $\mathscr{B} \cap \mathscr{D}$.

COROLlaRY 1. If in Theorem 1 the hypothesis (1.18) is replaced with

$$
u \geqslant 0 \text { on } \partial \mathscr{D}-(\Gamma \times\{0\}) \text { near } \Gamma \times\{0\},
$$

then the conclusion (1.19) can be replaced with

$$
u \geqslant 0 \text { on } \Gamma \times\{0\} .
$$

Proof. Apply Theorem 1 to each point $\left(y_{0}, 0\right)$ on $\Gamma \times\{0\}$.

3. Lateral points. Again we let $\mathscr{L}$ and $\mathscr{M}$ denote the operators defined by (*) and described in $\$ 2$. Condition (A) is replaced with the slightly stronger requirement that, for all $(x, t)$ in $\mathscr{D}$,

$$
\alpha|\xi|^{2} \leqslant \sum_{i, j=1}^{n} a_{i j}(x, t) \xi_{i} \xi_{j} \leqslant \beta|\xi|^{2}, \text { for all } \xi \text { in } \mathbf{R}^{n},
$$

where $\alpha$ and $\beta$ are positive constants, $0<\alpha \leqslant \beta$. We replace condition (B) by the more stringent

$$
|b(x, t)| \leqslant K
$$

for all $(x, t)$ in $\mathscr{D}$, for some nonnegative constant $K$. We retain the requirement that for all $(x, t)$ in $\mathscr{D}$,

$$
c(x, t) \leqslant 0 .
$$

For each $t$ in $(0, T)$ we may regard $t$ as fixed, and consider the operator $\mathscr{L}_{t}$ given by

$$
\mathscr{L}_{t}:=\sum_{i, j=1}^{n} a_{i j}(\cdot, t) \frac{\partial^{2}}{\partial x_{i} \partial x_{j}}+\sum_{i=1}^{n} b_{i}(\cdot, t) \frac{\partial}{\partial x_{i}}+c(\cdot, t),
$$

thereby obtaining a family $\left\{\mathscr{L}_{t}\right\}, t \in(0, T)$, of elliptic operators, with coefficients $a_{i j}(\cdot, t), b_{i}(\cdot, t), c(\cdot, t)$ real valued functions defined in $\Omega$. We discuss families of barrier functions associated with such a family of operators.

Definition. Let $\Gamma$ be a subset of $\partial \Omega$. Let $\left\{g_{y}\right\}, y \in \Gamma$, be a family of real valued functions, indexed by points on $\Gamma$, and let $\lambda$ be a real number. We say that $\left\{g_{y}\right\}$ is a family of uniformly strong local barriers of order $\lambda$ for the family $\left\{\mathscr{L}_{t}\right\}, t \in(0, T)$, in $\Omega$ at $\Gamma$ iff there exist positive constants $\rho, k_{1}, k_{2}, \ldots, k_{5}$ such that the following conditions hold (where we denote $g(x ; y):=g_{y}(x)$ ):

$\left(\mathrm{B}_{1}\right)$ Each $g_{y}$ is defined and continuous in $\bar{\Omega} \cap\{x: 0<|x-y|<\rho\}$, and of class $C^{2}$ in $\Omega \cap\{x: 0<|x-y|<\rho\}$.

$\left(\mathrm{B}_{2}\right)$ For $y$ on $\Gamma$, and $x$ in $\Omega$ with $0<|x-y|<\rho$,

$$
k_{1}|x-y|^{\lambda} \leqslant g(x ; y) \leqslant k_{2}|x-y|^{\lambda}
$$




$$
\begin{gathered}
\left|\frac{\partial g}{\partial x_{i}}(x ; y)\right| \leqslant k_{3}|x-y|^{\lambda-1}, \quad i=1, \ldots, n, \\
\left|\frac{\partial^{2} g}{\partial x_{i} \partial x_{j}}(x ; y)\right| \leqslant k_{4}|x-y|^{\lambda-2}, \quad i, j=1, \ldots, n, \\
\mathscr{L}_{t} g(x ; y) \leqslant-k_{5}|x-y|^{\lambda-2}, \quad t \in(0, T) .
\end{gathered}
$$

If $\lambda>0$, we say $\left\{g_{y}\right\}$ is a family of regular barriers, and, if $\lambda<0$, a family of singular barriers.

Note that for regular barriers, $g(x ; y) \rightarrow 0$ as $x \rightarrow y$, and for singular barriers, $g(x ; y) \rightarrow+\infty$ as $x \rightarrow y$. Both regular and singular barriers are positive in their region of definition except at $x=y$.

If $\Gamma$ consists of only a single point, say $y_{0}$, then $\left\{g_{y}\right\}, y \in \Gamma$, consists of only one function, say $g$. In this case we say that $g$ is a strong local barrier of order $\lambda$ for $\left\{\mathscr{L}_{t}\right\}$ in $\Omega$ at $y_{0}$.

Results of Miller [11, 12] for $n \geqslant 3$ and Oddson [13, 14] for $n=2$ imply the existence of families of strong regular and singular barriers. Although Miller and Oddson do not list explicitly (3.1)-(3.4) as properties of their barrier functions, an examination of their work shows that they have established the existence of families with these properties. Before discussing their results, we point out in the next lemma that, under appropriate boundedness conditions on the lower order coefficients of the family $\left\{\mathscr{L}_{t}\right\}$, a family of uniformly strong local barriers for the operators corresponding to the principal parts of $\left\{\mathscr{L}_{t}\right\}$ is an analogous family for $\left\{\mathscr{L}_{t}\right\}$. We define $\hat{\mathscr{L}}_{t}$ as the principal part of $\mathscr{L}_{t}$,

$$
\hat{\mathscr{L}}_{t}:=\sum_{i, j=1}^{n} a_{i j}(\cdot, t) \frac{\partial^{2}}{\partial x_{i} \partial x_{j}} .
$$

LEMMA 2-A. If $\left\{g_{y}\right\}, y \in \Gamma$, is a family of uniformly strong local barriers of order $\lambda$ for $\left\{\mathscr{L}_{t}\right\}$ in $\Omega$ at $\Gamma, t \in(0, T)$, then it also is for $\left\{\mathscr{L}_{t}\right\}$, provided that conditions $\left(\mathrm{B}^{*}\right),(\mathrm{C})$ on the first order coefficients of $\left\{\mathscr{L}_{t}\right\}$ hold.

Proof. We need only to check that, for some $\rho$ and positive $k_{5},(3.4)$ is valid for $0<|x-y|<\rho$, assuming that for some positive $\hat{k}, \hat{\rho}$, and for $0<|x-y|<\hat{\rho}$,

$$
\hat{\mathscr{L}}_{t} g(x ; y) \leqslant-\hat{k}|x-y|^{\lambda-2}, \quad t \in(0, T) .
$$

Writing

$$
\mathscr{L}_{t} g=\hat{\mathscr{L}}_{t} g+\sum_{i=1}^{n} b_{i}(\cdot, t) \frac{\partial g}{\partial x_{i}}+c(\cdot, t) g
$$

and using $\left(\mathrm{B}^{*}\right),(\mathrm{C}),(3.1),(3.2)$, we obtain

$$
\mathscr{L}_{t} g(x ; y) \leqslant-\hat{k}|x-y|^{\lambda-2}+n K k_{3}|x-y|^{\lambda-1} .
$$

Thus (3.4) holds for $0<|x-y|<\rho$ and some positive $k_{5}$ if $\rho$ is sufficiently small (independently of $y$ and $t$ ). 
Miller and Oddson proved the existence of strong barriers on cones for uniformly elliptic operators of principal part only,

$$
L:=\sum_{i, j=1}^{n} a_{i j} \frac{\partial^{2}}{\partial x_{i} \partial x_{j}}
$$

where the $a_{i j}$ 's are not necessarily continuous but satisfy a uniform ellipticity condition analogous to $\left(\mathrm{A}^{*}\right)$,

$$
\alpha|\xi|^{2} \leqslant \sum_{i, j=1}^{n} a_{i j}(x) \xi_{i} \xi_{j} \leqslant \beta|\xi|^{2} \quad(\beta \geqslant \alpha>0),
$$

for all $\xi$ in $\mathbf{R}^{n}$ and all $x$ in the interior of a cone $T_{\psi}$. The cone $T_{\psi}$, where $0<\psi<\pi$, lies in $\mathbf{R}^{n}$ with vertex at the origin, axis in the positive $x_{n}$-direction, and is defined explicitly as

$$
T_{\psi}=\{0\} \cup\left\{x \in \mathbf{R}^{n}: x \neq 0, \theta(x) \leqslant \psi\right\},
$$

with $\theta(x):=\operatorname{arc} \cos \left(x_{n} /|x|\right)$. Miller and Oddson were looking for slightly weaker barrier functions, requiring instead of (3.1) only that $g(x)>0$ in $T_{\psi}-\{0\}$, and instead of (3.4) only that $L g \leqslant 0$ in the interior of $T_{\psi}$. However the barrier functions that they produce are of the form $g(x)=|x|^{\lambda} F(\theta)$, and one can check that such functions $g$ also satisfy our stronger requirements for a strong barrier function. (This fact is pointed out by Miller in Theorem 3 of [12] for the case $\lambda>0$. For $\lambda<0$ a similar statement is valid.) Miller and Oddson in fact prove more than existence of barrier functions, showing further that for fixed $\alpha$ and $\beta$ there exist maximal and minimal orders of regular barriers and singular barriers, respectively, such that every elliptic operator consisting only of its principal part, with $\left(\mathrm{A}^{\dagger}\right)$ holding, has regular and singular barriers of orders arbitrarily close to these maximal and minimal orders, and regular barriers of orders arbitrarily close to zero. (Miller also proves a similar result for operators with lower order terms.) For dimension $n=2$ Oddson computes these maximal and minimal orders explicitly as functions of $\psi, \alpha$, and $\beta$. (Since these formulas are a little complicated we do not reproduce them here.) For $n \geqslant 3$ the computation of maximal and minimal orders of barriers on cones for uniformly elliptic operators appears to be an open problem.

A critical observation concerning the barrier functions of Miller and Oddson is that these functions depend only on the ellipticity constants $\alpha, \beta$ of $\left(\mathrm{A}^{\dagger}\right)$, and on the angular opening $\psi$ of the cone $T_{\psi}$, quantities which are invariant under translation and rotation of coordinates. Therefore these results can be applied to any point on our exceptional set $\Gamma$, provided that such a point is the vertex of a cone exterior to $\Omega$, and to any operator $\mathscr{L}_{t}, t \in(0, T)$. (We extend each $\mathscr{L}_{t}$ as the Laplace operator outside $\Omega$.) If each point on $\Gamma$ has the same size exterior cone, and if the bounds (A*) hold throughout $\Omega$, then we obtain in this manner a family of uniformly strong local barriers for the principal parts of $\left\{\mathscr{L}_{t}\right\}$. If moreover the lower order coefficients of $\left\{\mathscr{L}_{t}\right\}$ satisfy the bounds $\left(\mathrm{B}^{*}\right),(\mathrm{C})$, then Lemma $2-\mathrm{A}$ ensures that this family is also a family of uniformly strong local barriers, and of the same order, for $\left\{\mathscr{L}_{t}\right\}$. (From the proof of Lemma 2-A, one sees that the constant $K$ of (B*) affects 
the radius $\rho$ of definition of the barriers and also the constant $k_{5}$ of (3.4), but not the order of the barriers.)

So combining the results of Miller and Oddson with these observations, we have the following general statement concerning the existence of families of barriers:

Proposition 2. Suppose the family $\left\{\mathscr{L}_{t}\right\}, t \in(0, T)$, as defined by $(\dagger)$, has coefficients satisfying conditions $\left(\mathrm{A}^{*}\right),\left(\mathrm{B}^{*}\right),(\mathrm{C})$ for some nonnegative constants $\alpha, \beta$, and $K$. Let $\Gamma$ be a subset of $\partial \Omega$, and assume that, for each $y$ on $\Gamma, \Omega$ is contained inside a cone $C_{y}$ in $\mathbf{R}^{n}$ with vertex at $y$, and that moreover these cones $\left\{C_{y}\right\}, y \in \Gamma$, may all be chosen with the same angular opening $\theta$. Then there exists for $\left\{\mathscr{L}_{t}\right\}$ in $\Omega$ at $\Gamma$ families of uniformly strong local barriers, both regular and singular of orders $\lambda$ and $-\mu$, respectively, where $\lambda, \mu>0$, and $\lambda$ may be chosen arbitrarily close to zero. The possible orders $\lambda$ and $-\mu$ depend on $\alpha, \beta, K$ and on the common angular opening $\theta$ of the cones $\left\{C_{y}\right\}, y \in \Gamma$.

We can now state our basic result concerning exceptional boundary sets on the lateral boundary of $\mathscr{D}=\Omega \times(0, T)$.

THEOREM 3. Let $\mathscr{M}=\mathscr{L}-\partial / \partial t$ be given in $\mathscr{D}$ by $(*)$, with conditions $\left(\mathrm{A}^{*}\right),\left(\mathrm{B}^{*}\right)$, (C) holding, and let $\Gamma$ be a subset of $\partial \Omega$, with $y_{0}$ a point on $\Gamma$. Suppose there exists a family of uniformly strong local singular barriers of order $-\mu(\mu>0)$ for $\left\{\mathscr{L}_{t}\right\}$, $t \in(0, T)$, in $\Omega$ at $\Gamma$, and that there exists a strong local regular barrier of order $\lambda$ $(0<\lambda<2)$ for $\left\{\mathscr{L}_{t}\right\}$ in $\Omega$ at $y_{0}$. Suppose also that $\mathscr{H}(\Gamma)<\mu$, where $\mathscr{H}(\Gamma)$ is the Hausdorff dimension of $\Gamma$. Let $u$ be a real valued function in $\mathscr{D}=\Omega \times(0, T)$, of class $C^{2}$ with respect to $x$ and of class $C^{1}$ with respect to $t$. Suppose that for some nonnegative constant $M$ we have for $(x, t)$ in $\mathscr{D}$,

$$
u(x, t) \geqslant-M, \quad \mathscr{M} u(x, t) \leqslant M .
$$

Then for $0<t_{0}<T$, the boundary condition

$$
u \geqslant 0 \quad \text { on }(\partial \Omega-\Gamma) \times(0, T) \text { near }\left(y_{0}, t_{0}\right)
$$

implies that

$$
u \geqslant 0 \text { at }\left(y_{0}, t_{0}\right) .
$$

PROOF. Let $g=g\left(\cdot, y_{0}\right)$ be the strong local regular barrier of order $\lambda$ for $\left\{\mathscr{L}_{t}\right\}$ in $\Omega$ at $y_{0}$, and let $\left\{h_{y}\right\}, y \in \Gamma$, be the family of uniformly strong local singular barriers of order $-\mu$ for $\left\{\mathscr{L}_{t}\right\}$ in $\Omega$ at $\Gamma$. For simplicity of notation, we may assume that the constants $\rho, k_{1}, \ldots, k_{5}$ of (3.1)-(3.4) are the same for $\left\{h_{y}\right\}$ and $g$.

Condition (3.6) implies the existence of an open cylinder $\mathscr{C}$, described by

$$
\mathscr{C}:=\left\{(x, t) \in \mathbf{R}^{n+1}:\left|x-y_{0}\right|<r,\left|t-t_{0}\right|<\eta\right\},
$$

such that

$$
u \geqslant 0 \text { on }[(\partial \Omega-\Gamma) \times(0, T)] \cap \overline{\mathscr{C}} .
$$

We may choose the radius $r$ and height $2 \eta$ sufficiently small that

$$
r<\rho / 3, \quad\left(t_{0}-\eta, t_{0}+\eta\right) \subset(0, T) .
$$


Conditions (3.5), (3.4), (3.9), and $0<\lambda<2$ imply that in $\mathscr{C} \cap \mathscr{D}$,

$$
\mathscr{M} u(x, t)+\mathscr{M} g(x) \leqslant M-k_{5}\left|x-y_{0}\right|^{\lambda-2} \leqslant M-k_{5} r^{\lambda-2} .
$$

Thus we may choose $r$ smaller, if necessary, so that

$$
\mathscr{M}(u+g)(x, t)<-2 M / \eta \quad \text { in } \mathscr{C} \cap \mathscr{D} .
$$

We will establish (3.7) by showing that $\lim \inf u(x, t) \geqslant 0$ as $(x, t) \rightarrow\left(y_{0}, t_{0}\right)$ from inside $\mathscr{C} \cap \mathscr{D}$.

Define $\delta:=[\mu-\mathscr{H}(\Gamma)] / 2$; then $\delta>0$, and

$$
0 \leqslant \mathscr{H}(\Gamma)=\mu-2 \delta<\mu-\delta<\mu .
$$

Let $\nu$ be a positive constant, sufficiently small that

$$
0<\nu<r, \quad k_{1} \nu^{-\delta} \geqslant M
$$

Given $\varepsilon>0$, Lemma 1-C implies the existence of a countable covering of $\Gamma$ by open balls $\left\{B_{i}\right\}$ in $\mathbf{R}^{n}$ such that each center $y_{i}$ of $B_{i}$ lies on $\Gamma$, each radius $r_{i}$ of $B_{i}$ satisfies $0<r_{i} \leqslant \nu$, and moreover so that

$$
\sum_{i} r_{i}^{\mu-\delta}<\varepsilon
$$

We may discard all balls in this collection not meeting $\Gamma \cap\left\{x \in \mathbf{R}^{n}:\left|x-y_{0}\right|<r\right\}$, so that $\left\{B_{i}\right\}$ covers $\Gamma \cap\left\{x:\left|x-y_{0}\right|<r\right\}$ and (3.12) continues to hold. Next we define a function $v$ according to

$$
\begin{aligned}
v(x, t):= & u(x, t)+\left(1+\frac{M}{k_{1} r^{\lambda}}\right) g(x) \\
& +\sum_{i} r_{i}^{\mu-\delta} h\left(x ; y_{i}\right)+\frac{M}{\eta^{2}}\left(t-t_{0}\right)^{2} .
\end{aligned}
$$

For $(x, t)$ in $\mathscr{C} \cap \mathscr{D}$ and each $i$,

$$
\left|x-y_{i}\right| \leqslant\left|x-y_{0}\right|+\left|y_{0}-y_{i}\right| \leqslant r+\left(r+r_{i}\right) \leqslant 2 r+\nu<3 r<\rho,
$$

and moreover $\left|x-y_{0}\right| \leqslant r<\rho$; hence all $h\left(x ; y_{i}\right)$ and $g\left(x ; y_{0}\right)$ are defined and of class $C^{2}$ as functions of $(x, t)$ in $\mathscr{C} \cap \mathscr{D}$. For any $(x, t)$ in $\mathscr{C} \cap \mathscr{D}$ we also have, for all $i$,

$$
0<\operatorname{dist}(x, \Gamma) \leqslant\left|x-y_{i}\right|<\rho .
$$

Using these inequalities, (3.12), and the bounds (3.1)-(3.3) (with $g$ replaced by $h$ and $\lambda$ by $-\mu$ ), we see that the series in (3.13) and the differentiated series up to order two converge uniformly on compact subsets of $\mathscr{C} \cap \mathscr{D}$; thus the function $v$ of (3.13) is of class $C^{2}$ in $x$ and of class $C^{1}$ in $t$ in $\mathscr{C} \cap \mathscr{D}$, with termwise differentiation up to these orders being allowed. From (3.13), (3.10), and (3.4) (for both $g$ and $h$ ), we conclude that in $\mathscr{C} \cap \mathscr{D}$,

$$
\begin{aligned}
\mathscr{M} v(x, t) & \leqslant \mathscr{M} u(x, t)+\mathscr{M} g(x)-\frac{2 M}{\eta^{2}}\left(t-t_{0}\right) \\
& \leqslant \mathscr{M}(u+g)(x, t)+\frac{2 M}{\eta}<0 .
\end{aligned}
$$


Now we define open sets $\hat{\mathscr{C}}$ and $\mathscr{P}$ in $\mathbf{R}^{n+1}$;

$$
\hat{\mathscr{C}}:=\mathscr{C} \cap \mathscr{D}, \quad \mathscr{P}:=\left(\bigcup_{i} B_{i}\right) \times \mathbf{R} .
$$

Since $\operatorname{dist}(x, \Gamma)>0$ for $(x, t)$ in $\mathscr{C} \cap \mathscr{D}$, and $\operatorname{dist}(x, \Gamma) \leqslant\left|x-y_{i}\right| \leqslant r_{i} \leqslant \nu$ for $(x, t)$ in $B_{i} \times \mathbf{R}$, the open set $\hat{\mathscr{C}}-\overline{\mathscr{P}}$ is nonempty if $\nu$ is sufficiently small; in fact, any point $(x, t)$ in $\hat{\mathscr{C}}$ will lie in $\hat{\mathscr{C}}-\overline{\mathscr{P}}$ if $\nu<\operatorname{dist}(x, \Gamma)$. We will show that $v \geqslant 0$ in $\hat{\mathscr{C}}-\overline{\mathscr{P}}$ by applying the minimum principle to $v$ in this open set.

As in the proof of Theorem 1, the boundary of $\hat{\mathscr{C}}-\overline{\mathscr{P}}$ can be divided into

$$
\partial(\hat{\mathscr{C}}-\overline{\mathscr{P}}) \subset(\partial \mathscr{C} \cap \overline{\mathscr{D}}-\mathscr{P}) \cup(\mathscr{C} \cap \partial \mathscr{D}-\mathscr{P}) \cup(\hat{\mathscr{C}} \cap \partial \mathscr{P}) .
$$

(i) On $\partial \mathscr{C} \cap \overline{\mathscr{D}}-\mathscr{P}$, we have either (a) $\left|x-y_{0}\right|=r$, or (b) $\left|t_{0}-t\right|=\eta$. In case (a), (3.13), (3.5), (3.1), and $\left|t_{0}-t\right| \leqslant \eta$ give

$$
v(x, t) \geqslant-M+\frac{M}{k_{1} r^{\lambda}} k_{1}\left|x-y_{0}\right|^{\lambda}=0,
$$

and in (b), (3.13), (3.5), (3.1) give

$$
v(x, t) \geqslant-M+M \eta^{2} / \eta^{2}=0 .
$$

(ii) On $\mathscr{C} \cap \partial \mathscr{D}-\mathscr{P}$, we have $v \geqslant 0$ by (3.8) and the inclusion $(\mathscr{C} \cap \partial \mathscr{D}-\mathscr{P}) \subset$ $[(\partial \Omega-\Gamma) \times(0, T)] \cap \overline{\mathscr{C}}$.

(iii) On $\hat{\mathscr{C}} \cap \partial \mathscr{P}$, we have $\operatorname{dist}(x, \Gamma)>0$. Since $r_{i} \rightarrow 0$ by (3.12), $x \in \bar{B}_{i}$ for at most a finite number of $i$, and hence for some positive integer $N$,

$$
x \in \partial\left(\bigcup_{i=1}^{N} B_{i}\right) \subset \bigcup_{i=1}^{N}\left(\partial B_{i}\right),
$$

which implies $x \in \partial B_{j}$ for some $j$. But then, by (3.13), (3.5), (3.1), (3.11), and $r_{j} \leqslant \nu$,

$$
\begin{aligned}
v(x, t) & \geqslant u(x, t)+r_{j}^{\mu-\delta} h\left(x ; y_{j}\right) \geqslant-M+r_{j}^{\mu-\delta} k_{1}\left|x-y_{j}\right|^{-\mu} \\
& =-M+k_{1} r_{j}^{-\delta} \geqslant-M+k_{1} \nu^{-\delta} \geqslant 0 .
\end{aligned}
$$

We have now shown that $\mathscr{M} v<0$ in $\mathscr{C} \cap \mathscr{D}$ and $v \geqslant 0$ on

$$
\partial(\hat{\mathscr{C}}-\overline{\mathscr{P}})=\partial(\mathscr{C} \cap \mathscr{D}-\overline{\mathscr{P}}) .
$$

By the minimum principle for parabolic operators, we have $v \geqslant 0$ in $\mathscr{C} \cap \mathscr{D}-\overline{\mathscr{P}}$. By (3.1) and (3.12), the series in (3.13) can be bounded in $\mathscr{C} \cap \mathscr{D}$ by

$$
\sum_{i} r_{i}^{\mu-\delta} h\left(x ; y_{i}\right) \leqslant \sum_{i} r_{i}^{\mu-\delta} k_{2}\left|x-y_{i}\right|^{-\mu} \leqslant \varepsilon k_{2}[\operatorname{dist}(x, \Gamma)]^{-\mu} ;
$$

hence from $v \geqslant 0$ it follows that in $\mathscr{C} \cap \mathscr{D}-\overline{\mathscr{P}}$,

$$
u(x, t)+\left(1+\frac{M}{k_{1} r^{\lambda}}\right) g(x)+\frac{M}{\eta^{2}}\left(t-t_{0}\right)^{2} \geqslant-\varepsilon k_{2}[\operatorname{dist}(x, \Gamma)]^{-\mu} .
$$

We let $\nu \rightarrow 0^{+}$to shrink $\mathscr{P}$, with (3.11) being preserved; since $\varepsilon$ is arbitrary we obtain that in $\mathscr{C} \cap \mathscr{D}$,

$$
u(x, t)+\left(1+\frac{M}{k_{1} r^{\lambda}}\right) g(x)+\frac{M}{\eta^{2}}\left(t-t_{0}\right)^{2} \geqslant 0 .
$$


Since $\lim \inf g(x)=0$ as $x \rightarrow y_{0}$ by (3.1), we obtain $\lim \inf u(x, t) \geqslant 0$ as $(x, t) \rightarrow$ $\left(y_{0}, t_{0}\right)$ in $\mathscr{C} \cap \mathscr{D}$.

COROLlaRy 3. If in Theorem 3 the hypothesis (3.6) is replaced with

$$
u \geqslant 0 \text { on }(\partial \Omega-\Gamma) \times(0, T) \text { near } \Gamma \times(0, T),
$$

then the conclusion (3.7) can be replaced with

$$
u \geqslant 0 \text { on } \Gamma \times(0, T) \text {. }
$$

Proof. Apply Theorem 3 to each point $\left(y_{0}, t_{0}\right)$ on $\Gamma \times(0, T)$.

As a sample consequence of Theorem 3 and its corollary, we specialize to the case when the principal part of $\mathscr{L}$ is the Laplace operator $\Delta$; then

$$
\mathscr{M} u=\Delta u+\sum_{i=1}^{n} b_{i} \frac{\partial u}{\partial x_{i}}+c u-\frac{\partial u}{\partial t} .
$$

Proposition 4. Suppose the principal part of $\mathscr{L}$ is $\Delta$, as in (3.16), and assume $\left(\mathrm{B}^{*}\right)$ and $(\mathrm{C})$ continue to hold in $\mathscr{D}=\Omega \times(0, T)$. Let $\Gamma$ be a subset of $\partial \Omega$ such that for each $y$ on $\Gamma, \Omega$ is contained on one side of an $(n-1)$-dimensional hyperplane passing through $y$. Suppose also that the Hausdorff dimension of $\Gamma$ is less than $n-1$. If $u$ is a real valued function in $\mathscr{D}$, of class $C^{2}$ in $x$ and class $C^{1}$ in $t$, satisfying, for some nonnegative constant $M$,

$$
u(x, t) \geqslant-M, \quad \mathscr{M} u(x, t) \leqslant M
$$

for all $(x, t)$ in $\mathscr{D}$, then $u \geqslant 0$ on $(\partial \Omega-\Gamma) \times(0, T)$ near $\Gamma \times(0, T)$ implies $u \geqslant 0$ on $\Gamma \times(0, T)$.

Proof. It follows from results of [7] that the hyperplane condition implies the existence of uniformly strong local barriers of order $-\mu$ for $\left\{\mathscr{L}_{t}\right\}$ in $\Omega$ at $\Gamma$, for any $\mu$ in the range $0<\mu<n-1$. The condition also implies the existence of regular barriers. Thus Theorem 3 and its corollary can be applied.

\section{REFERENCES}

1. H. S. Bear and G. N. Hile, Behaviour of solutions of elliptic differential inequalities near a point of discontinuous boundary data, Comm. Partial Differential Equations 8 (1983), 1175-1197.

2. P. C. Fife, Growth and decay properties of solutions of second order elliptic equations, Ann. Scuola Norm. Sup. Pisa 20 (1966), 675-701.

3. A. Friedman, On two theorems of Phragmen-Lindelof for linear elliptic and parabolic differential equations of the second order, Pacific J. Math. 7 (1957), 1563-1575.

4. D. Gilbarg, The Phragmén-Lindelöf Theorem for elliptic partial differential equations, J. Rational Mech. Anal. 1 (1952), 411-417.

5. K. Habetha, Zum Phragmén-Lindelöf Prinzip bei partiellen Differentialgleichungen, Arch. Math. 15 (1964), 324-331.

6. J. O. Herzog, Phragmen-Lindelöf theorems for second order quasilinear elliptic partial differential equations, Proc. Amer. Math. Soc. 15 (1964), 721-728.

7. G. N. Hile and R. Z. Yeh, Phragmén-Lindelöf principles for solutions of elliptic differential inequalities, J. Math. Anal. Appl. 107 (1985), 478-497.

8. Exceptional boundary sets for solutions of elliptic partial differential equations, Indiana Univ. Math. J. 35 (1986), 611-621.

9. E. Hopf, Remarks on the preceding paper of D. Gilbarg, J. Rational Mech. Anal. 1 (1952), 419-424. 
10. P. D. Lax, A Phragmén-Lindelöf theorem in harmonic analysis and its application to some questions in the theory of elliptic equations, Comm. Pure Appl. Math. 10 (1957), 361-389.

11. K. Miller, Barriers on cones for uniformly elliptic operators, Ann. Mat. Pura Appl. 76 (1967), 93-105.

12. __ Extremal barriers on cones with Phragmén-Lindelöf theorems and other applications, Ann. Mat. Pura Appl. 90 (1971), 297-329.

13. J. K. Oddson, Some solutions of elliptic extremal equations in the plane, Matematiche (Catania) 23 (1968), 273-289.

14. Phragmén-Lindelöf theorems for elliptic equations in the plane, Trans. Amer. Math. Soc. 145 (1969), 347-356.

15. M. H. Protter and H. F. Weinberger, Maximum principles in differential equations, Prentice-Hall, Englewood Cliffs, N. J., 1967.

16. J. B. Serrin, On the Phragmén-Lindelöf principle for elliptic differential equations, J. Rational Mech. Anal. 3 (1954), 395-413.

Department of Mathematics, University of HaWaII, Honolulu, HaWAII 96822 